\title{
CUTTING METAL SHEET WITH A PUNCH WITH INTERNAL HEATING
}

This paper describes issues related to the process of cutting with a punch with internal heating. The theoretical part explains the effects related to thermal expansion, conduction and transfer of heat in the body of punches. The experimental part refers to the design of a tool with an internally heated punch. Experimental results are summarised and the process is analysed in the discussion.

Keywords: Metal sheet, internal heating, cutting, cutting tool, forming.

\section{Introduction}

Forming technologies have long used various ways and methods based primarily on physical knowledge. For a longer time, the method has been used of deep drawing using heating the active parts of drawing tools. The first person to describe this method was Romanovskij in 1968 (p. 191, and he published the process picture No. 114 B) [1]. In 1965, Srp, et al. published this solution (p. 132, Figure 199) [2]. This method is also presented by Cabelka, et al. in 1967 (pp. 261-262, Figure 3.509) [3], Blascik, et al. in 1988 (pp. 297-298, Figure 1298) [4] and Baca, in 2000 (p. 131, Figure 108) [5]. This paper originated on the basis of the above information. We will discuss the possibility of using internal heating in metal sheet cutting using a cutting machine. In the past we have already published a paper on the propagation of heat in the cut zone, in which we dealt with this issue - Moravec in 2000 [6].

\section{Theoretical part}

\section{First approximation of the problem}

If a cylindrical cutting punch is considered a rod clamped at one end, then a first approximation can read as follows: rods, when heated, expand especially in length. When heating, the amplitudes of oscillating molecules increase and the molecules fill a larger space. Therefore, solids stretch in all directions. A cylinder-shaped cutting punch can be considered the surface area $S_{l}$, which is the area prior to a change in temperature. When performing calculations, we can understand surface expansion as linear expansion in two directions/dimensions (Fig. 1) [7].

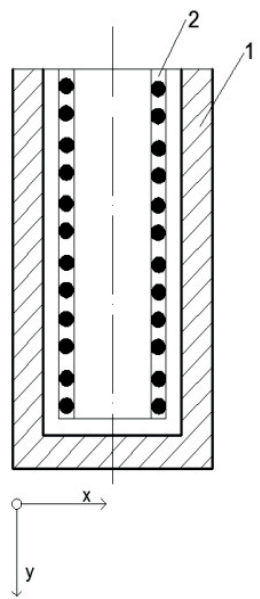

Fig. 1 Scheme of a cutting punch with internal heating 1 - punch body, 2 - heating body,

The variables are as follows: $S_{1}$ - the surface area prior to temperature change, $S_{2}$ - the surface area following temperature change, $\Delta S$ - the surface area difference $=S_{2}-S_{1,} \Delta t$ - the temperature difference $=t_{2}-t_{1}$.

The following also applies: $\Delta S=S_{2}-S_{1}=l_{2}^{2}-l_{1}^{2}=l_{1}^{2}\left(1-\alpha \Delta t^{2}\right)-l_{1}^{2}=l_{1}^{2}\left[1+2 \alpha \Delta t+\alpha^{2}(\Delta t)^{2}\right]-l_{1}^{2}$ Since $a$ is small, the member with the square number in the equation can be neglected. Then, the following will apply: $\Delta \mathrm{S}=l_{2}^{2}(1+2 \alpha \Delta t)-l_{1}^{2}=l_{1}^{2} 2 \alpha \Delta \mathrm{t}$, and also: $\Delta \mathrm{S}=S_{1} 2 \alpha \Delta t$,

\footnotetext{
* Jan Moravec

Department of Technological Engineering, Faculty of Mechanical Engineering, University of Zilina, Slovakia

E-mail: jan.moravec@fstroj.uniza.sk
} 
as well as: $S_{2}=S_{I}+\Delta S=S_{I}+S_{1} 2 \alpha \Delta t^{2}$, and finally: $S_{2}=S_{1}\left(1+2 a \Delta t^{2}\right)$.

Note: The coefficient $\alpha$ depends on temperature to a very limited extend only. Within the range 0 to $100{ }^{\circ} \mathrm{C}$, the values apply as given in physical tables, and with sufficient accuracy.

\section{Heat conduction and transfer in a rod}

The rod is made of a mono-material, and is of a constant cross-section. The base of the rod has heat conducted in a way that at all points of the initial cross-section the temperature $T_{a}$ (Fig. 2) is the same. The rod is situated in an environment with the same unchanging temperature $T_{0}$. Assuming that the material thermal conductivity coefficient as well as the heat transfer coefficient are constant all across the rod surface, we are to determine temperature distribution over the rod length, and determine heat transfer [ 8 and 9].

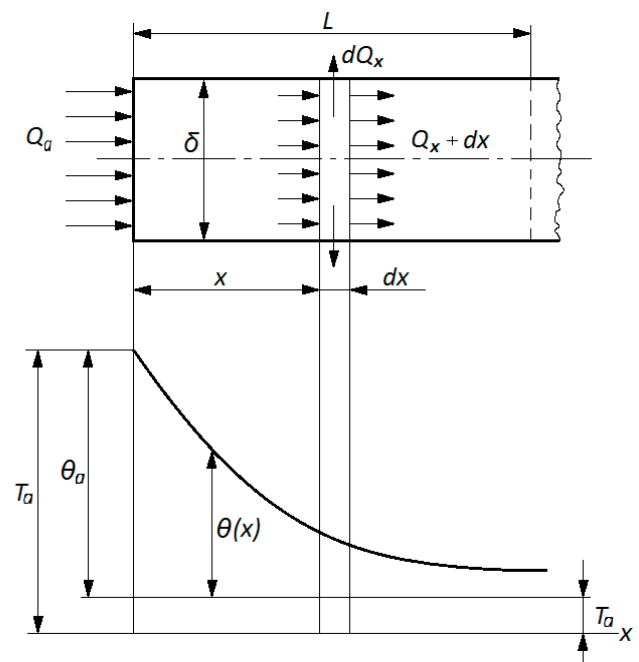

Fig. 2 Diagram for determining heat conduction and transfer through the rod

Solving can be done assuming a one-dimensional heat flow. This assumption will be all the closer to reality, the lower will be

the following values:
Biot number for a rod $\left(\left(B i \equiv \frac{\alpha \delta}{\lambda} \ll 1\right), \alpha\right.$ is the heat transfer coefficient from the rod surface into the environment; $\hat{o}-$ the rod thickness; $\lambda-$ the thermal conductivity coefficient of the rod material).

Biot number represents the ratio of values $\frac{\alpha}{\lambda / \delta}$, which are characteristic of the intensity of heat removal from the rod surface into the environment, compared to heat input intensity to the rod surface from the rod material. The lower the Biot number, the lower heat removal from the cooled body surface, compared to a possible heat supply from the inside. Due to the above facts, for $B i$ « 1 we can approximately consider, when solving heat conduction in a rod, that the temperature gradient in the rod cross section be sufficiently small compared to the temperature gradient along the rod axis. Using this assumption/condition, we can execute the equation of rod heat conduction equation solving as a task with one-dimensional heat flux.

We execute an elementary layer of the thickness $d x$, and for this rod thickness we write an equation of heat balance. Heat flux in the elementary layer changes under stationary conditions due to the heat transfer on the outer surface:

$Q_{x}=Q_{x}+d x+d Q_{x} \quad$ or $\quad S \lambda \frac{d^{2} \Theta}{d x^{2}} d x=u \cdot d x \cdot \alpha \cdot \Theta$,

where $\theta=T(x)-T_{0}$ is the temperature difference between the rod and the environment.

In this way we arrive to a differential equation of heat conduction along a rod:

$\frac{d^{2} \Theta}{d x^{2}}-m^{2} \Theta=0$

where $\frac{d^{2} \Theta}{d x^{2}}-m^{2} \Theta=0$ is the equation's dimensional
parameter.

The integral of this differential equation is in the form: $\Theta(x)=C_{1} e^{+m x}+C_{2} e^{-m x}$.

\section{Experimental part}

A cavity is formed inside the cutting punch body. A heating body is placed into the cavity. The heating body heats the face of the cutting punch. It was necessary to find out what parameters correspond to the required temperature. A $40 \mathrm{~W}$ tiny heating body (device) from a micro-solder proved to be suitable for experimental work. As shown in Fig. 3, we connected the assembly to a 230V source, which ensured heating of the active part of the cutting punch. Figure 4 shows a view of the experimental workplace of temperature sensing. The ambient temperature was $26{ }^{\circ} \mathrm{C}$. We found out the following: at $U=116 \mathrm{~V}$, and after 25 minutes, we achieved the temperature of $97{ }^{\circ} \mathrm{C}$, and after 40 minutes the temperature increased to $112{ }^{\circ} \mathrm{C}$ - the value that had to be obtained (to be more precise - we needed to achieve temperature between 100 and $110{ }^{\circ} \mathrm{C}$ ). The real temperature in experimental conditions varied between 108 and $113{ }^{\circ} \mathrm{C}$. We chose this temperature to avoid initiation of phase transformations. The voltage $U_{\text {term }}=4.435 \mathrm{mV} \rightarrow t=110^{\circ} \mathrm{C}, U_{\text {term }}=4.280 \mathrm{mV} \rightarrow t=105.7$ ${ }^{\circ} \mathrm{C} U_{\text {term }}=4.404 \mathrm{mV} \rightarrow t=109{ }^{\circ} \mathrm{C}$. The final values were as follows: $U=116 \mathrm{~V}$, current $I=78 \mathrm{~mA}, U_{\text {term }}=4.404 \mathrm{mV}$. 


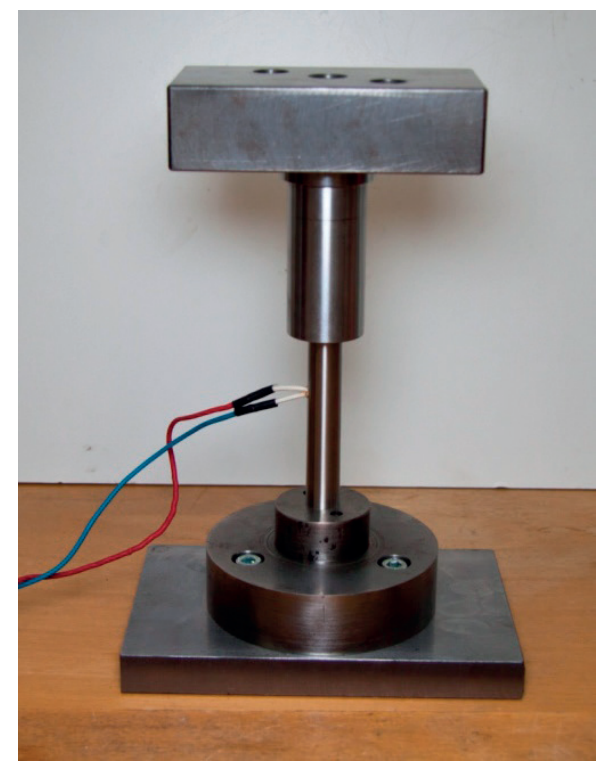

Fig. 3 Assembly of the experimental cutting tool

Prior to heating, the cutting punch diameter was $15.91 \mathrm{~mm}$, and after heating it reached $15.922 \mathrm{~mm}$, which was measured directly and does not differentiate significantly from the calculated value. This laboratory verification was followed by incorporation of the modified cutting punch with internal heating into the cutting tool assembly. Since it is an open tool without any cutting punch guidance, we had to precisely adjust the tool and consistently clamp it on the press. Figures 5 and 6 show the experimental cutting tool and its clamping on the press.

The presented tool was clamped on a LESP 63 press, and used to cut $1-\mathrm{mm}$ metal sheet. The metal sheet was made of conventional STN 411321 material, with the composition of $0.10 \% \mathrm{C}, 0.45 \% \mathrm{Mn}, 0.035 \% . P, 0.035 \% \mathrm{~S}$. The other values included $R_{m}=420 \mathrm{MPa}, R_{e}=235 \mathrm{MPa}, A=26 \%$. The number of blanks was chosen in thousands of pieces. The cutting punch was heated to $108^{\circ} \mathrm{C}\left( \pm 5^{\circ}\right)$. From this perspective, we can assess the cutting process with heating as stable. Attention was focused on the effect of heating during the cutting process.

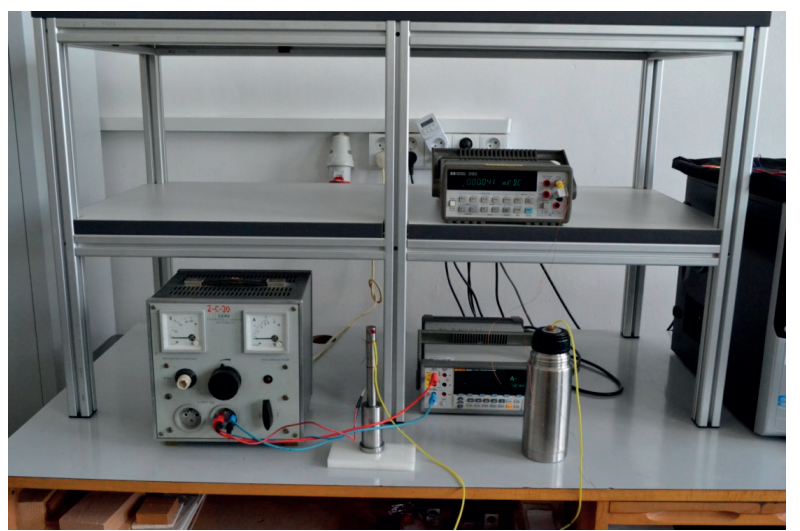

Fig. 4 Experimental workplace

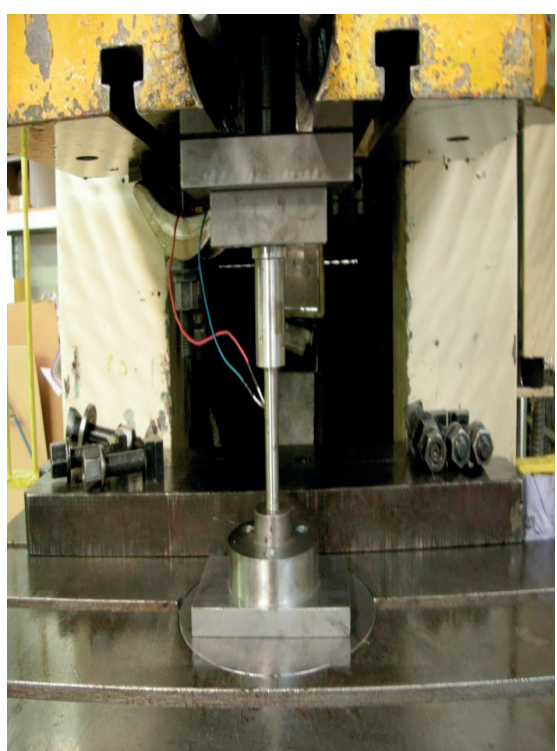

Fig. 5 Workplace for cutting

\section{Technological aspect}

Using a heated cutting punch, we identified some factors in experimental work that are instrumental in deeper understanding of the above issues. The condition is that heating does not replace the forming tool sharpening, alignment or repair, but only helps prolong the phase of economic wear (Fig. 6). The size of the production batch is of significant influence in the manufacture of blanks (Fig. 7) [10, 11 and 12].

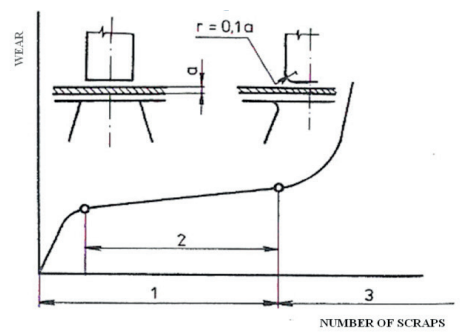

Fig. 6 Criterion of the cutting edge wear 1 - sharp tool, 2 - economic wear, 3 - supercritical wear

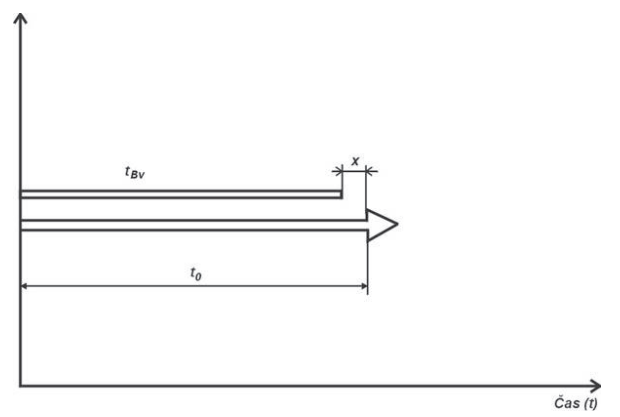

Fig. 7 Optimum distribution of continuous production time and tool operation time 
Figure 7 shows the optimum distribution of the continuous blank production time and the quality tool operation. The difference $x$ enables re-sharpening and re-aligning of the tool in this section of time value difference. Therefore, it is important to determine the optimum size of the production batch, whereas it should apply that during the time of manufacturing a certain batch, the tool should ensure the quality of parts manufactured in the given batch:

$t_{B v} \leq t_{o}$,

where:

$t_{B v}$ - the time for manufacturing a batch with $n$ pieces, $t_{o}=t_{p n}$ the time of quality operation of the tool between two acts of re-sharpening or adjustment.

Condition: the degree of precision of the manufacturing process is directly dependent on the quality and precision of the forming tool.

When applying a heated punch in the process of metal sheet cutting, we have to consider what can be achieved with this solution. The following applies generally to sheet cutting, which is a common operation in the preparation of blanks, such as bending or deep drawing: cutting tolerance plays a major role. This tolerance is directly dependent on the thickness and mechanical properties of the material being cut. Cutting gap $(C G)$ is half the cutting tolerance. Optimal $C G$ is an important measure of quality of the cutting tool, and it contributes significantly to the overall quality of the desired result of the cutting process.

The geometry of punches and punching dies includes the shape, dimensions and tolerances of the working parts of the cutting tools. The following applies: the size of the punched hole determines the punch dimensions, and the blank size determines the punching die and punch dimensions. In hole-punching operations (openings and blanks), the part of material that falls from the opening (waste) determines the size of the cut-out hole, whereas the size of the punch and punching die is enlarged by the cutting tolerance. The size of a blank resulting from the cutting operation depends on the punching die size, therefore the cutting gap size is on the expense of the cutting punch. The cutting punch size will be smaller by the cutting tolerance value. Hence, it follows that the cutting tolerance is the difference in dimensions of the punching die and the punch in the corresponding point of cross-section. It is the distance of the punch from the punching die after its insertion at any point. The ideal cutting gap is uniform all along the punching die (punch) circumference, and is equal to half the cutting tolerance (Fig. 8).

Optimum cutting gap is an important measure of quality of cutting tools. It has a decisive influence on the durability of the cutting edge and the service life of the tool. Its size depends mainly on the thickness and mechanical properties of the material to be cut. An optimum cutting gap is considered the one that is uniform along the entire cut curve and that achieves the required quality of the cutting surface with a minimum exercised force and labour. A reduction in the cutting gap leads to an increase in cutting force as well as labour. Increase in the force is not large, but increase in labour can reach up to $40 \%$.

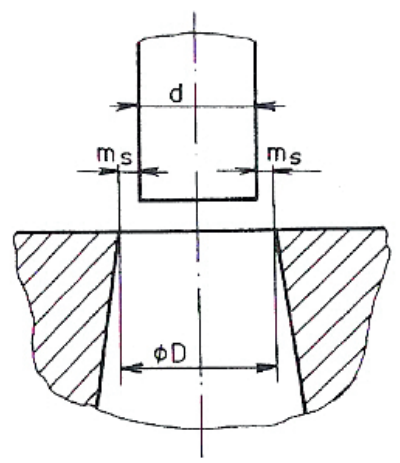

Fig. 8 Cutting gap and cutting tolerance

Since the size of the cutting gap changes during the operation due to the cutting edge wear, new tools are made with minimum acceptable cutting gap. Cutting edges gradually wear out, the cutting gap grows, and when it exceeds the region of economic wear, the forming force increases by $55 \%$. It applies for the upper limit of the economic wear region that the cutting edge radius $R=0.1 a$, where $a$ is the thickness of the material being cut (Fig. 6). Such wear is followed by a necessary renovation of cutting edges [13 and 14].

However, the cutting gap size can be determined also theoretically from the following formula:

$m_{s}=\left(a-h_{v}\right) \operatorname{tg} \beta$

where:

$h_{v}$ - the indentation depth; $h_{v} \approx(0.2$ to 0.5$) a$,

$\beta$ - the cutting surface angle relative to the direction of the cutting force acting, which has the following values according to the properties of the material being cut: mild (soft) steel $\beta=5$ to $6^{\circ}$, medium-hard steel $\beta=4$ to $5^{\circ}$, and hard steel $\beta=4^{\circ}$.

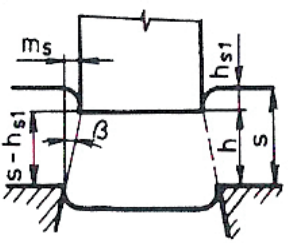

Fig. 9 Determining the cutting gap size 
According to Fig. 9, the punching die profile is characterised by relief grinding (clearance) by the angle $\alpha$. This profile is used in blanks with lower shape accuracy requirements and in larger metal sheet thickness.

It follows from the above considerations that using a heated punch possibly results in quite properly corrected size of the cutting tolerance. As mentioned above, the increase in applied force at reduced cutting gap is small, but the labour increment is significant. This can be easily read in the graph in Fig. 6 . Therefore, we are dealing with the maximum length of section 2 that represents the economic wear. The amount of labour in this section increases rather gradually, not in leaps. Certainly, there is also a significant impact of the amount of heating of the active part of the cutting tool.

When applying a heated cutting punch, one must consider such a structure as early as in the design stage of the forming tool, with the economic aspect of the process coming to the fore here. According to Fig. 10, the appropriate cutting tolerance is exceeded at a certain stage of the cutting process. And it is exactly at this moment that a heated cutting punch can be applied so that the acting part of the forming tool gets heated and quite simply corrects the cutting tolerance in order to ensure that the process runs as if with a re-adjusted tool and corrected cutting tolerance. Using this solution can be generally recommended in the cases when the cutting tolerance increases to the extent that the blanks feature visible burrs with a height exceeding the permissible level of cut tolerance. The size (dimensions) of cutting punches also plays its role here, since the larger the size, the larger the linear expansion coefficient. Of course, the important in the process is only the direction in the $x$ axis, elongation in the $y$ direction does not affect the cutting process (Fig. 1).
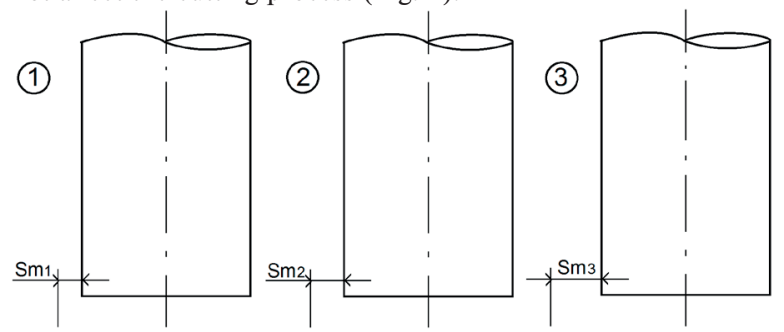

Fig. 10 Change in the cutting gap resulting from the cutting punch wear

$$
s_{m 1}<s_{m 2}>s_{m 3}
$$

\section{Quality of cutting surface}

Field tests did not find any significant difference on the cutting surfaces between the quality of the surface being cut traditionally (without any heating), and with the applied heating of the active part. The above facts had been expected, and the assumption was fully confirmed. Surface roughness measurement was carried out using MITUTOYO Surftest. The surface roughness was $R_{a}=0.77$ $\mu \mathrm{m}$ (Fig. 11).

\section{Discussion}

Heat conduction and transfer in a hollow cylinder with heat sources

In a hollow cylinder body there are evenly distributed heat sources with a specific output $q_{z d r}\left[\mathrm{~W} / \mathrm{m}^{3}\right]$. The wall of the cylinder transfers heat from the inside and the outside to the environment that is of unchanging (in time) temperatures $T_{1}$ and $T_{2}$. To solve this problem we need to use the following boundary conditions:

For internal heat exchange at $r=d_{l} / 2$ :

$$
T_{d_{1} / 2}=-\frac{q_{z d r}}{\lambda} \frac{1}{4}\left(\frac{d_{1}}{2}\right)^{2}+C_{1} \ln \frac{d_{1}}{2}+C_{2} .
$$

Due to the boundary condition:

$$
-\left.\frac{d T}{d r}\right|_{r=d_{1} / 2}=-\frac{\alpha_{1}}{\lambda}\left(T_{d_{1} / 2}-T_{1}\right)
$$

the equation is:

$$
\begin{aligned}
& -\frac{q_{z d r}}{\lambda} \frac{1}{2} \frac{d_{1}}{2}+C_{1} \frac{1}{d_{1} / 2}= \\
& -\frac{\alpha_{1}}{\lambda}\left[C_{2}+C_{1} \ln \frac{d_{1}}{2}-\frac{q_{z d r}}{\lambda} \frac{1}{4}\left(\frac{d_{1}}{2}\right)^{2}-T_{1}\right]
\end{aligned}
$$

For external heat exchange at $r=d_{2} / 2$ :

$$
T_{d_{2} / 2}=-\frac{q_{z d r}}{\lambda} \frac{1}{4}\left(\frac{d_{2}}{2}\right)^{2}+C_{1} \ln \frac{d_{2}}{2}+C_{2} .
$$

Due to the boundary condition of the third kind:

$$
-\left.\frac{d T}{d r}\right|_{r=d 2 / 2}=-\frac{\alpha_{2}}{\lambda}\left(T_{d_{2} / 2}-T_{2}\right)
$$

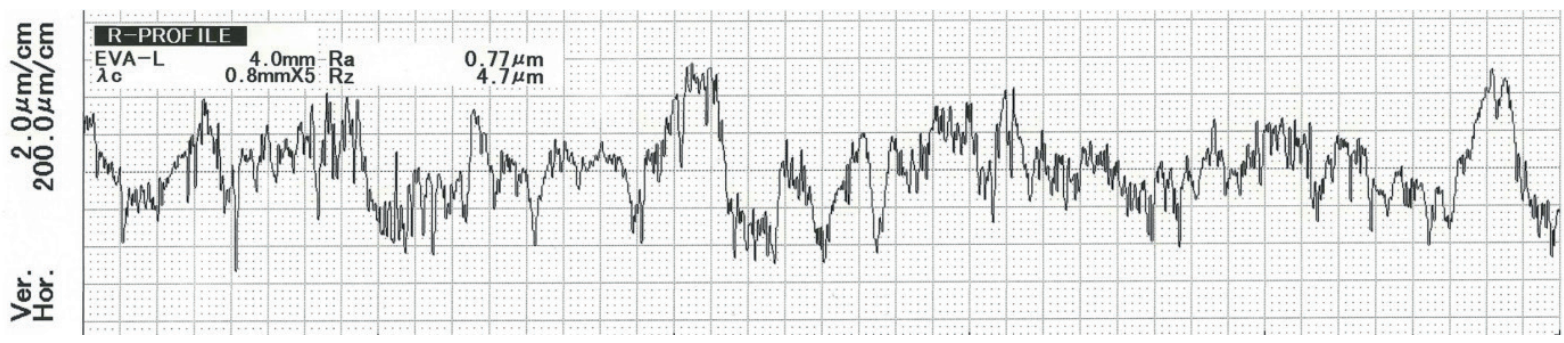

Fig. 11 Surface roughness when hot cutting after 850 strokes 
the equation takes the following form:

$$
\begin{aligned}
& -\frac{q_{z d r}}{\lambda} \frac{1}{2} \frac{d_{2}}{2}+C_{1} \frac{1}{d_{2} / 2}= \\
& -\frac{\alpha_{2}}{\lambda}\left[C_{2}+C_{1} \ln \frac{d_{2}}{2}-\frac{q_{z d r}}{\lambda} \frac{1}{4}\left(\frac{d_{2}}{2}\right)^{2}-T_{2}\right]
\end{aligned}
$$

Using the two related equations of boundary conditions we determine the constants $C_{1}$ and $C_{2}$ :

$C_{1}=\frac{B_{1} \alpha_{2}+B_{2} \alpha_{1}}{A_{1} \alpha_{2}+A_{2} \alpha_{1}}$

$C_{2}=\frac{A_{1} B_{2}-A_{2} B_{1}}{A_{1} \frac{\alpha_{2}}{\lambda}+A_{2} \frac{\alpha_{1}}{\lambda}}$,

$A_{1}=\frac{1}{d_{1} / 2}+\frac{\alpha_{1}}{\lambda} \ln \frac{d_{1}}{2}$,

$A_{2}=\frac{1}{d_{2} / 2}+\frac{\alpha_{2}}{\lambda} \ln \frac{d_{2}}{2}$,

$B_{1}=\frac{q_{z d r}}{\lambda} \frac{1}{2} \frac{d_{1}}{2}\left(1-\frac{\alpha_{1}}{\lambda} \frac{1}{2} \frac{d_{1}}{2}\right)-\frac{\alpha_{1}}{\lambda} T_{1}$,

$B_{2}=\frac{q_{z d r}}{\lambda} \frac{1}{2} \frac{d_{2}}{2}\left(1-\frac{\alpha_{2}}{\lambda} \frac{1}{2} \frac{d_{2}}{2}\right)-\frac{\alpha_{2}}{\lambda} T_{2}$

The equation of temperature distribution in the cylindrical wall is in the following form:

$T(r)=-\frac{q_{z d r}}{\lambda} \frac{1}{4} r^{2}+C_{1} \ln r+C_{2}$.

The total amount of heat $Q$ transferred from the inside and the outside cylinder surfaces to the environment, related to the cylinder length unit, is determined as the sum of:

$Q=-\left.\pi d_{2} \lambda \frac{d T}{d r}\right|_{r=\frac{d_{2}}{2}}+\left.\pi d_{1} \lambda \frac{d T}{d r}\right|_{r=\frac{d_{1}}{2}}=$

$\pi d_{2} \lambda\left(\frac{q_{z d r}}{\lambda} \frac{1}{2} \frac{d_{2}}{2}-C_{1} \frac{2}{d_{2}}\right)-\pi d_{1} \lambda\left(\frac{q_{z d r}}{\lambda} \frac{1}{2} \frac{d_{1}}{2}-C_{1} \frac{2}{d_{1}}\right)$

and after the adjustment it will be:

$Q=\frac{\pi}{4}\left(d_{2}^{2}-d_{1}^{2}\right) q_{z d r} \quad[\mathrm{~W} / \mathrm{m}]$, i. e. the total heat transfer is equal to the aggregate source output in the cylinder volume.

\section{Conclusion}

This paper describes the issue of applying heating to the cutting process. We presented the theoretical background, construction solution (design) of an experimental tool, and the results of experimental tests. The discussion delivered an insight into the issue of using metal sheet cutting with heating the active component. Phase transformation is each qualitative change in the state of the same substance (in particular, steel), which occurs at least in a certain part of the observed system due to changes in conditions (temperature, pressure, electric field, magnetic field). The above processes may after all result in complete disappearance of the initial matrix phase, to be replaced by a new phase. In the case of an active component such cutting punch, this actually becomes disabled since the required hardness is reduced. In thermo-mechanical terms, phase transformations constitute a transition from the state when it is characterised by a higher value of free enthalpy to a state with its lower value. The loss of energy is represented by labour involved in carrying out the contemplated conversion:

- $\Delta G=\left(d A_{\max }\right)_{\text {neob }}$

where: $\Delta G=\Delta H-T \Delta S,\left(d A_{\max }\right)_{\text {nеobj }}$ is the maximum non-volume labour, $\Delta H$ is the system enthalpy change, $T$ is the temperature $(\mathrm{K}), \Delta S$ is the system enthalpy change [15].

This paper adds new knowledge and conclusions to the large sphere of the issue of metal sheet cutting.

The article was created in frame of VEGA 1/0551/14.

\section{Acknowledgements}

Hereby we would like to thank Mr. Prof. RNDr. P. Bury, Head of Department of Technical Physics, and Mr. F. Cernobil for facilitating the execution of laboratory tests. Our gratitude also goes to Mr. Ing. B. Melo, PhD of the Vienna International, spol. s r. o., Martin, for facilitating the execution of operational tests in their press shop.

\section{References}

[1] ROMANOVSKIJ, V. P.: Guide for Cold Pressing (in Czech), 1968, SNTL-SVTL : Praha.

[2] SRP, K. et al.: Basics of Pressing (in Czech), 1965, SNTL : Praha.

[3] CABELKA, J. et al.: Mechanical Technology (in Slovak), SAV : Bratislava, 1967. 
[4] BLASCIK, F. et al.: Forming Technology, Casting and Welding (in Slovak), ALFA : Bratislava, 1988.

[5] BACA, J.: Forming (in Slovak), SVST : Bratislava, 2000.

[6] MORAVEC, J. : Heat Transfer Zone Shear (in Slovak), Materialove inzinierstvo - Material Engineering, vol. 7, No. 2,2000, pp. 29-37, ISSN 1335-0803.

[7] MORAVEC, J. : Unconventional Methods of Forming Metal (in Slovak),EDIS : University of Zilina, 2011, 135 p., ISBN 978-80-8070554-0233-8

[8] HOSFORD, W. F., CADDEL, R. M.: Metal Forming, Mechanics and Metallurgy, Cambridge University Press, 2011, ISBN 978-1-10700452-8.

[9] JOHNSON, W., MAMALIS, A. G.: Plasticity and Metal Forming, 1998, Cambridge,

[10] MARCINIAK, Z., DUNCAN, J. L., HU, S. J.: Mechanics of Sheet Metal Forming, Butterworth-Heinemann, Jordan Hill : Oxford, 2002, p. 221, ISBN 0-7506-5300-9.

[11] BOLJANOVIC, V.: Sheet Metal Forming Processes and die Design, 2005, ISBN 08311-3182-9.

[12] BILIK, J.: Forming Technology (in Slovak), STU: Bratislava, 2000.

[13] PEARCE, R.: Sheet Metal Forming, 2010, ISBN 0-7503-0101-5.

[14] SPISAK, E. et al.: Engineering Technology (in Slovak), 2011, ISBN 978-80-553-08-203.

[15] BELAN, J.: Theory of Phase Transformations Selected Chapters (in Slovak), 2015, EDIS : University of Zilina, ISBN 978-80-2241034-0. 